\title{
SELF-CONSISTENT SIZE AND VELOCITY DISTRIBUTIONS OF COLLISIONAL CASCADES
}

\author{
Margaret Pan ${ }^{1}$ and Hilke E. Schlichting ${ }^{2,3,4}$ \\ ${ }^{1}$ Department of Astronomy, University of California, Berkeley, CA 94720, USA; mpan @ astro.berkeley.edu \\ ${ }^{2}$ Department of Earth and Space Sciences, University of California, Los Angeles, CA 90095, USA; hilke@ ucla.edu \\ ${ }^{3}$ Theoretical Astrophysics, MC 350-17, California Institute of Technology, Pasadena, CA 91125, USA \\ Received 2011 November 1; accepted 2012 January 3; published 2012 February 22
}

\begin{abstract}
The standard theoretical treatment of collisional cascades derives a steady-state size distribution assuming a single constant velocity dispersion for all bodies regardless of size. Here we relax this assumption and solve self-consistently for the bodies' steady-state size and size-dependent velocity distributions. Specifically, we account for viscous stirring, dynamical friction, and collisional damping of the bodies' random velocities in addition to the mass conservation requirement typically applied to find the size distribution in a steady-state cascade. The resulting size distributions are significantly steeper than those derived without velocity evolution. For example, accounting self-consistently for the velocities can change the standard $q=3.5$ power-law index of the Dohnanyi differential size spectrum to an index as large as $q=4$. Similarly, for bodies held together by their own gravity, the corresponding power-law index range $2.88<q<3.14$ of Pan \& Sari can steepen to values as large as $q=3.26$. Our velocity results allow quantitative predictions of the bodies' scale heights as a function of size. Together with our predictions, observations of the scale heights for different-sized bodies for the Kuiper belt, the asteroid belt, and extrasolar debris disks may constrain the mass and number of large bodies stirring the cascade as well as the colliding bodies' internal strengths.
\end{abstract}

Key words: Kuiper belt: general - minor planets, asteroids: general - planetary systems - protoplanetary disks

Online-only material: color figures

\section{INTRODUCTION}

Believed to be a primary mechanism operating in circumstellar dusty debris disks as well as our own Kuiper and asteroid belts, collisional cascades - the transfer of mass from larger to smaller sized bodies via collisions between those bodies-are ubiquitous in our Galaxy. Their widespread occurrence and potential importance in understanding planet formation and planet-disk interactions have naturally motivated considerable study. Theoretical treatments predicting the collisional size distribution have included analytic work as well as numerical simulations. The pioneering treatment of Dohnanyi (1969), who analytically calculated the size distribution for a steady-state cascade of constant-strength bodies, has been elaborated upon, extended to size-dependent strength laws, and applied to different physical contexts by several authors, including Williams \& Wetherill (1994), Tanaka et al. (1996), O'Brien \& Greenberg (2003, 2005), Kenyon \& Bromley (2004), Pan \& Sari (2005), and Löhne et al. (2008). Some of these also considered non-power-law features in the size distribution such as waves due to the gravity-strength transition (O'Brien \& Greenberg 2003, 2005; Pan \& Sari 2005) or changes in the fragment size spectrum (Belyaev \& Rafikov 2011). Many numerical studies of collisional cascades have also been performed (see, for example, Campo Bagatin et al. 1994; Durda \& Dermott 1997; Kenyon \& Bromley 2004, 2008; Krivov et al. 2005; Löhne et al. 2008; Wyatt 2008; Fraser \& Kavelaars 2009).

The analytic and most of the numerical works on collisional cascades have generally assumed that the bodies' velocity dispersion is independent of size and constant in time once the cascade begins. Nevertheless, it is not obvious why we would expect constant size-independent velocities in a typical cascade. Indeed, processes like viscous stirring and collisional damping should affect all bodies' velocities at size-dependent

\footnotetext{
${ }^{4}$ Hubble Fellow.
}

rates throughout the cascade's lifetime. Because the velocities directly affect the frequencies of catastrophic collisions, deviations from the constant velocities typically assumed could significantly change previous results on size distributions in steady-state cascades. When extrapolated over many orders of magnitude in size-for example, between $\sim$ kilometersized planetesimals and $\sim$ millimeter-sized or smaller dust particles - even small inaccuracies in the equilibrium size distribution can mean large errors in the expected number of bodies and/or in the bodies' internal strengths as inferred from size distribution observations.

Here we incorporate velocity evolution processes into our treatment of collisional cascades and find the size spectrum and size-dependent velocity dispersion self-consistently. In Section 2 we give expressions for the physical processes operating in the cascade. The well-known mass conservation condition is the basis of previous work beginning with Dohnanyi (1969), so we summarize it briefly in Section 2.1. In Section 2.2 we describe the stirring and damping processes affecting the velocities, and in Section 2.3 we give expressions for the velocity equilibrium required in a steady-state cascade. These are the velocity analogs of the mass conservation conditions of Section 2.1. In Section 3 we impose mass conservation and velocity equilibrium together to find the size and velocity power laws of steady-state cascades. As we explain, in a disk of bodies with a power-law size distribution we expect to see up to three different velocity regimes. We derive velocity and size power laws in all three regimes for both gravity- and strengthdominated bodies. In Section 4 we compare our analytic results to those of our fragmentation simulations. Finally, in Section 5 we summarize and discuss our findings.

\section{SIZE AND VELOCITY EVOLUTION PROCESSES}

In order to find the size distribution and velocity function selfconsistently, we assume a debris disk that occupies an annulus 
with typical orbital angular frequency $\Omega$. The bodies in the cascade have uniform composition and body mass density $\rho$, and their sizes $r$ cover the range $\left[r_{\min }, r_{\max }\right]$. We write the differential body size spectrum as $d N / d r \propto r^{-q}$ and the velocity function as $v(r) \propto r^{p}$. The number of bodies of size larger than or equal to $r$ is then $N(r) \propto r^{1-q}$. Because typically $q>1, N(r)$ is also of order the number of bodies in a logarithmic size interval about $r$, and we will use these two meanings interchangeably. We consider in turn how mass conservation in the cascade and velocity evolution via gravitational stirring and collisional damping constrain $q$ and $p$. Since our primary goal is to clearly delineate the relevant physical processes, we work to order of magnitude throughout.

\subsection{Mass Conservation}

We begin with mass conservation, the basis for most analytic cascade treatments to date. Our discussion of mass conservation parallels that of Pan \& Sari (2005), and we refer readers to that work for a more detailed description. In a steady-state collisional cascade where mass is conserved in catastrophic collisions, the total mass destroyed per unit time per logarithmic interval in radius must be independent of size. This implies

$$
\begin{aligned}
\text { constant } & =\rho r^{3} \cdot N(r) \cdot \frac{N\left(r_{B}(r)\right)}{\text { volume }} \cdot r^{2} \cdot v_{\text {rel }} \\
& =\rho r^{3} \cdot N(r) \cdot \frac{N\left(r_{B}(r)\right) r^{2} \Omega}{\text { area }},
\end{aligned}
$$

where $r_{B}(r)$ is the size of the smallest body, or bullet, that can destroy a target of size $r$ in a collision and $v_{\text {rel }}$ is the typical relative velocity of bullets and targets. The second line follows because we assume isotropic velocities, so that the scale height of the disk is of order $v_{\text {rel }} / \Omega$. The volume of Equation (1) in which the bodies move is the area occupied by the disk midplane times this scale height, so the mass conservation relation for a disk is independent of velocity.

We further assume that the way the bodies break is independent of size, that is, the shape of the average fragment size distribution is size-independent. We parameterize the bullet-target size relation as a power law $r_{B}(r) \propto r^{\alpha}$. Then Equation (2) yields

$$
q=\frac{6+\alpha}{1+\alpha}
$$

The value of $\alpha$ depends on how much energy is lost in the post-impact destruction process. In the gravity regime, we can think of the destruction as a shock induced in the target by the bullet which propagates to the antipode of the impact site. The limiting cases for the shock propagation are energy conservation and momentum conservation in the shocked material; these give respectively

$$
\begin{aligned}
& \rho r^{3} v_{\mathrm{esc}}^{2}(r) \sim \rho r_{B}^{3} v^{2}(r) \quad \longrightarrow \alpha=(5-2 p) / 3 \\
& \rho r^{3} v_{\mathrm{esc}}(r) \sim \rho r_{B}^{3} v(r) \quad \longrightarrow \alpha=(4-p) / 3 .
\end{aligned}
$$

Here, we assumed $p \geqslant 0: p<0$ would in principle require arbitrarily large velocities for arbitrarily small sizes, so we will not consider that case here. Numerical simulations of catastrophic collisions find $1.37<\alpha<1.66$ with constant collision velocities (see, for example, Stewart \& Leinhardt 2009; Benz \& Asphaug 1999, and references therein); this is consistent with the range $4 / 3<\alpha<5 / 3$ for $p=0$ which we find above. Together, Equations (3)-(5) imply

$$
\frac{22-p}{7-p}>q>\frac{23-2 p}{8-2 p} .
$$

The inequalities hold if $p<1$, which as we will see in Section 3 is satisfied.

In the strength regime, $\alpha$ depends on the material properties of the body, which are often parameterized as $Q^{*}(r)$, the energy per unit mass needed to destroy a body of size $r$. In the strength regime simulations find $Q^{*}(r) \propto r^{\gamma}$ where $0 \geqslant \gamma>-1 / 2$ (Benz \& Asphaug 1999; Stewart \& Leinhardt 2009). Then

$$
\rho r^{3} Q^{*}(r)=\rho r_{B}^{3} v^{2}(r) \quad \longrightarrow \quad \alpha=1+\frac{\gamma-2 p}{3}
$$

where, again, we assume $p \geqslant 0$. With Equation (3) this gives

$$
q=\frac{21+\gamma-2 p}{6+\gamma-2 p}
$$

As an example, Dohnanyi (1969) used in effect $\gamma=p=0$ in the strength regime; these immediately yield $q=7 / 2$ in Equation (8).

\subsection{Velocity Evolution Processes}

We now consider velocity evolution. Physically, $v(r)$ depends on stirring from larger bodies and damping from collisions with and dynamical friction from smaller bodies. In the following, we explore the stirring-damping balance in detail. Motivated by observations of the asteroid and Kuiper belts, we work in the regime where the typical relative velocity in an encounter between two bodies is larger than either body's Hill velocity, which is of order a body's escape velocity times the one-sixth power of the ratio of the body's mass and the central mass.

We begin by writing expressions for the rates at which viscous stirring, collisional damping, and dynamical friction damping affect a body of size $r$. Our treatment of velocity evolution is similar to that given in Goldreich et al. (2004), and we refer readers to that work for a clear and detailed description of the stirring and damping rate expressions. The rate at which bodies are viscously stirred by bodies of size $R \geqslant r$ depends on which of them is moving faster. If $v_{\text {esc }}(R)>v(R)>v(r)$, the rate is

$$
\left.\frac{1}{v(r)} \frac{d v(r)}{d t}\right|_{\text {stir }} \sim \frac{N(R) R^{2} \Omega}{\text { area }}\left(\frac{v_{\mathrm{esc}}(R)}{v(R)}\right)^{2}\left(\frac{v_{\mathrm{esc}}(R)}{v(r)}\right)^{2} .
$$

This focusing factor applies because we need only double $v(r)$, not necessarily $v(R)$. If $v(R)<v(r)<v_{\text {esc }}(R)$, we have

$$
\left.\frac{1}{v(r)} \frac{d v(r)}{d t}\right|_{\mathrm{stir}} \sim \frac{N(R) R^{2} \Omega}{\text { area }}\left(\frac{v_{\mathrm{esc}}(R)}{v(r)}\right)^{4} .
$$

Because $v_{\text {esc }}(R) \propto R$, the largest bodies in the system do most of the stirring unless $q>5$ when $v(R)>v(r)$ or unless $q>7$ when $v(R)<v(r)$. We expect these conditions to hold in real systems, so we will assume them throughout. We will show in Section 3 that this assumption is self-consistent in the cascade.

The rate at which bodies of size $r$ are damped by direct collisions with bodies of size $s \leqslant r$ is

$$
\left.\frac{1}{v(r)} \frac{d v(r)}{d t}\right|_{\text {damp }} \sim \frac{N(s) \Omega}{\text { area }} \frac{s^{3}}{r} .
$$


There is no focusing factor here because, as we discuss later, dynamical friction damping is faster than damping by direct collisions only if $v(r) \leqslant v_{\text {esc }}(r) .{ }^{5}$ Note that bodies in the cascade must have $v(r) \geqslant v_{\mathrm{esc}}(r)$ because catastrophic collisions would otherwise be impossible. Equation (11) implies that if $q>4$, collisional damping is dominated by the smallest bodies in the disk, giving $s=r_{\text {min }}$.

When $q \leqslant 4$, collisions between equal-sized bodies dominate, ${ }^{6}$ and Equation (11) becomes

$$
\left.\frac{1}{v(r)} \frac{d v(r)}{d t}\right|_{\text {damp }} \sim \frac{N(r) \Omega}{\text { area }} r^{2} .
$$

However, in the cascade $r_{B}(r) \leqslant r$. Then the collisional destruction rate will be at least as fast as the damping rate of Equation (12) as long as $q>1$; the rates are equal only when $r_{B}(r)=r$. Simulations of catastrophic collision ejecta indicate that nearly all the kinetic energy relative to the bullet-target center of mass is lost to heat in a catastrophic collision (see, for example, Jutzi et al. 2010). Then bodies whose bullet-target mass ratio is not too small should lose most of their velocity dispersion in a catastrophic collision. If we assume that destructive collisions do indeed provide significant damping,

$$
\left.\left.\frac{1}{v(r)} \frac{d v(r)}{d t}\right|_{\text {damp }} \sim \frac{1}{v(r)} \frac{d v(r)}{d t}\right|_{\text {coll }} \sim \frac{N\left(r_{B}(r)\right) r^{2} \Omega}{\text { area }} .
$$

Note that in this case "significant damping" means that the largest collision fragments' typical random velocities are much less than those of the original targets. If instead destructive collisions cannot damp effectively - that is, the largest fragment retains most of its pre-collision velocity-then whether or not collisional cooling is effective depends on the age of the disk. Bodies of size $r$ are collisionally cooled only if the disk age is longer than the collision timescale implied by Equation (12).

The dynamical friction damping rate of size $r$ bodies by size $s<r$ bodies also depends on whether $v(r)>v(s)$ or vice versa. By analogy to the expressions of Equations (9) and (10) for viscous stirring, the two expressions for dynamical friction are

$$
\begin{aligned}
& \left.\frac{1}{v(r)} \frac{d v(r)}{d t}\right|_{\mathrm{df}} \\
& \sim\left\{\begin{array}{l}
\frac{N(s) \Omega}{\operatorname{area}} \frac{s^{3}}{r}\left(\frac{v_{\mathrm{esc}}(r)}{v(s)}\right)^{2}\left(\frac{v_{\mathrm{esc}}(r)}{v(r)}\right)^{2} \\
v(s)<v(r)<v_{\mathrm{esc}}(r) \\
\frac{N(s) \Omega}{\operatorname{area}} \frac{s^{3}}{r}\left(\frac{v_{\mathrm{esc}}(r)}{v(s)}\right)^{4} \\
v(r)<v(s)<v_{\mathrm{esc}}(r) .
\end{array}\right.
\end{aligned}
$$

As mentioned above, dynamical friction acts faster than direct collisions by $v_{\mathrm{esc}}^{4}(r) /(v(s) v(r))^{2}$ if $v(r)>v(s)$ or by $\left(v_{\text {esc }}(r) / v(s)\right)^{4}$ if $v(r)<v(s)$, so it applies to bodies with $v(r)<v_{\text {esc }}(r)$ which have not entered the cascade. The dynamical friction damping rate scales as $s^{4-q-2 p}$ if $v(r)>v(s)$ and as $s^{4-q-4 p}$ if $v(r)<v(s)$. Then the smallest bodies with $s=r_{\text {min }}$ dominate the damping if $q+2 p>4$ when $v(r)>v(s)$ or if $q+4 p>4$ when $v(r)<v(s)$.

\footnotetext{
5 Indeed, if $v(r) \geqslant v_{\mathrm{esc}}(r)$, dynamical friction is equivalent to elastic direct collisions.

6 The $q=4$ size spectrum is a marginal case in which collisions with bodies of all sizes should contribute equally to the damping. Since this represents only an order unity correction to the damping rate, the scalings given remain valid.
}

\subsection{Velocity Equilibrium}

With expressions in hand for rates of velocity evolution, we can impose the steady-state condition that stirring and damping balance. In addition to the size and velocity power laws $q, p$ of bodies in the cascade, we consider the analogous power laws $q^{\prime}, p^{\prime}$ for any bodies of size $r>r_{\max }$ which may be present in the disk but are too large to have entered the cascade. If the bodies in the disk formed through core accretion, we would expect $1<q^{\prime}<5$ (see, for example, Kenyon \& Bromley 2004, 2008; Schlichting \& Sari 2011) as well as the $q<5$ we already assumed. Motivated by the discussion after Equation (10), we let the largest bodies in the disk have size $R$.

We first consider bodies in the cascade. As explained in Section 2.2, these bodies are viscously stirred and collisionally damped. For cascades in which catastrophic collisions damp velocities effectively, velocity equilibrium means

$$
\begin{gathered}
0=\left.\frac{1}{v(r)} \frac{d v(r)}{d t} \sim \frac{1}{v(r)} \frac{d v(r)}{d t}\right|_{\mathrm{stir}}-\left.\frac{1}{v(r)} \frac{d v(r)}{d t}\right|_{\mathrm{coll}} \\
\sim\left\{\begin{array}{l}
\frac{N(R) R^{2} \Omega}{\operatorname{area}}\left(\frac{v_{\mathrm{esc}}(R)}{v(r)}\right)^{2}\left(\frac{v_{\mathrm{esc}}(R)}{v(R)}\right)^{2}-\frac{N\left(r_{B}(r)\right) r^{2} \Omega}{\operatorname{area}} \\
\frac{N(r)<v(R)<v_{\mathrm{esc}}(R)}{\operatorname{area}}\left(\frac{v_{\mathrm{esc}}(R)}{v(r)}\right)^{2}-\frac{N\left(r_{B}(r)\right) r^{2} \Omega}{\operatorname{area}} \\
v_{\mathrm{esc}}(R)>v(r)>v(R)
\end{array}\right.
\end{gathered}
$$

where we have applied Equations (9), (10), and (13). Equivalently, if $v(R)>v(r)$ the ratio of stirring and collision rates is

$$
\begin{aligned}
1 \sim & \frac{N(R)}{N\left(r_{B}(r)\right)}\left(\frac{R}{r}\right)^{2}\left(\frac{v_{\mathrm{esc}}(R)}{v(R)}\right)^{2}\left(\frac{v_{\mathrm{esc}}(R)}{v(r)}\right)^{2} \\
\sim & \left(\frac{r_{\mathrm{max}}}{r}\right)^{\alpha(1-q)+2+2 p} \\
& \cdot \frac{N(R)}{N\left(r_{B}\left(r_{\max }\right)\right)} \frac{R^{2}}{r_{\max }^{2}}\left(\frac{v_{\mathrm{esc}}(R)}{v(R)}\right)^{2}\left(\frac{v_{\mathrm{esc}}(R)}{v\left(r_{\max }\right)}\right)^{2}
\end{aligned}
$$

and if $v(R)<v(r)$ this ratio is

$$
1 \sim\left(\frac{r_{\max }}{r}\right)^{\alpha(1-q)+2+4 p} \cdot \frac{N(R)}{N\left(r_{B}\left(r_{\max }\right)\right)} \frac{R^{2}}{r_{\max }^{2}}\left(\frac{v_{\mathrm{esc}}(R)}{v\left(r_{\max }\right)}\right)^{4} .
$$

Note that we have transferred the coefficient of $r^{\alpha(1-q)}$ in $N\left(r_{B}(r)\right)$ to $N\left(r_{B}\left(r_{\max }\right)\right)$ in the last step. In Equations (18) and (19), the second through last terms on the right-hand side are simply the ratio of the stirring and collision rates for size $r_{\text {max }}$ bodies, those at the top of the cascade: compare them, for example, to the right-hand side of Equation (17), which is the ratio of stirring and collision rates for size $r$ bodies. This indicates that if the stirring and collision rates for size $r_{\max }$ bodies balance-which we expect since these bodies have just entered the cascade - the rest of the cascade will also be in velocity equilibrium if

$$
\begin{array}{ll}
q=1+\frac{2+2 p}{\alpha} & v(R)>v(r) \\
q=1+\frac{2+4 p}{\alpha} & v(R)<v(r) .
\end{array}
$$


In the gravity regime, using Equations (4) and (5) together with Equation (20) gives

$$
\begin{array}{ll}
\frac{10+5 p}{4-p}>q>\frac{11+4 p}{5-2 p} & v(R)>v(r) \\
\frac{10+11 p}{4-p}>q>\frac{11+10 p}{5-2 p} & v(R)<v(r) .
\end{array}
$$

The inequalities for $q$ hold when $-1 / 2<p<1$, which as we will see in Section 3 is satisfied. Similarly, in the strength regime we use Equation (7) with Equation (20) to get

$$
\begin{array}{lll}
q=\frac{9+\gamma+4 p}{3+\gamma-2 p} & v(R)>v(r) \\
q=\frac{9+\gamma+10 p}{3+\gamma-2 p} & v(R)<v(r) .
\end{array}
$$

We next consider a disk in which catastrophic collisions do not damp the velocities. This may occur, for example, if $r_{B}(r) \ll r$, in which case the center-of-mass velocity of a colliding bullet-target pair is dominated by the target velocity. Then conservation of momentum dictates that the velocity of the largest collisional fragment will be quite similar to the target's velocity even if all of the relative kinetic energy between the bullet and target is lost. If $q \leqslant 4$ and if the system's lifetime is at least as long the timescale for two bodies of size $r_{\text {max }}$ to collide, damping occurs through collisions between equal-sized bodies according to Equation (12). This damping mechanism dominates for all bodies with $v(r)>v_{\mathrm{esc}}(r)$. While this condition holds over the entire cascade, it may hold for bodies outside the cascade as well. To see that all bodies in the cascade are included, note that if $v(r)<v_{\mathrm{esc}}(r)$, then the impact energy in a collision between equal-sized bodies, $\sim \rho r^{3} v^{2}(r)$, is less than the gravitational binding energy $\sim \rho r^{3} v_{\text {esc }}^{2}(r)$ of either body. If $p>0$, the impact energy in a collision with a smaller bullet is likewise less than the gravitational binding energy of the target. Since both gravity- and strength-dominated bodies require impact energy at least as large as their gravitational binding energies, $v(r)>v_{\mathrm{esc}}(r)$ is required in the cascade. However, $v(r)>v_{\text {esc }}(r)$ may also apply for some bodies larger than $r_{\text {max }}$.

A calculation entirely analogous to that of Equations (16)(20) above which uses damping by Equation (12) rather than Equation (13), and the size where $v(r) \sim v_{\mathrm{esc}}(r)$ instead of $r_{\max }$, gives

$$
\begin{aligned}
& q=3+2 p \quad v(R)>v(r) \\
& q=3+4 p \quad v(R)<v(r) .
\end{aligned}
$$

If $q>4$, we substitute Equation (11) for Equation (12) in the above calculation. In this case $N(r)$ disappears from the ratio of stirring and damping rates and we get a condition on $p$ alone:

$$
\begin{aligned}
& p=1 / 2 \quad v(R)>v(r) \\
& p=1 / 4 \quad v(R)<v(r) .
\end{aligned}
$$

If the cascade lifetime is short compared to the timescale for collisions between bodies of size $r_{\max }$, then some bodies near the top of the cascade will not have had time to damp. For these undamped bodies, we expect shallower velocity power laws.

Finally, we consider any bodies in the disk whose velocities are smaller than their escape velocities. They cannot be part of the cascade, so we expect them to have sizes $r>r_{\max }$. The equilibrium velocities of these bodies follow from a balance between viscous stirring and dynamical friction. If $v(r)>v(R)$, we equate the stirring rate of Equation (9) with the damping rates of Equation (14) to get

$$
\begin{array}{ll}
\frac{v(r)}{v(s)} \sim\left(\frac{N(R)}{N(s)}\right)^{1 / 2} \frac{R^{3}}{s^{3 / 2} r^{3 / 2}} & v(s)<v(r) \\
\frac{v(r)}{v(s)} \sim\left(\frac{N(R)}{N(s)}\right)^{1 / 4} \frac{R^{3 / 2}}{s^{3 / 4} r^{3 / 4}} & v(s)>v(r) .
\end{array}
$$

Here, $s$ is the size of bodies which dominate the dynamical friction. Because we have broken power-law size and velocity distributions, and because the power-law breaks do not occur at $r$, we expect $s$ to be independent of $r$. If $v(r) \propto r^{p^{\prime}}$, Equation (25) implies

$$
\begin{array}{ll}
p^{\prime}=-3 / 2 & v(s)<v(r) \\
p^{\prime}=-3 / 4 & v(s)>v(r) .
\end{array}
$$

This is indeed consistent with the $v(r)>v(R)$ we assumed. Note that the kinetic energy per body, $\sim \rho r^{3} v^{2}(r) \propto r^{3+2 p^{\prime}}$, cannot increase with decreasing body size, so these bodies lie outside the cascade. For the same reason we can neglect any dynamical friction heating effects, which contribute at most an order unity correction.

If instead we assume $v(r)<v(R)$ and replace Equation (9) with Equation (10) above, no self-consistent solution for $p^{\prime}$ is possible.

\section{STEADY-STATE SIZE AND VELOCITY DISTRIBUTIONS}

We now solve simultaneously the mass conservation and velocity stirring/damping balance conditions of Section 2 to find the steady-state size and velocity distributions in the disk. We first confirm that the steady-state condition-equivalent to requiring that $N\left(r_{\max }\right)$ changes on a timescale long compared to collisions between and stirring of smaller bodies-is physical. Since the stirring cross-section of size $r$ bodies scales as $r^{-2 p}$, smaller bodies are indeed stirred faster than the largest bodies break. Similarly, since smaller bodies have more total surface area than larger bodies as long as $q>3$, smaller bodies break faster than larger ones. Our assumption of a steady state is therefore reasonable for all bodies smaller than $r_{\max }$. Said another way, $r_{\max }$ corresponds to the location of the break seen in collisional size distributions separating collisional and primordial bodies (O'Brien \& Greenberg 2003; Kenyon \& Bromley 2004; Pan \& Sari 2005). As bodies of size $r_{\max }$ break and $N\left(r_{\max }\right)$ decreases, the normalization of the cascade below $r_{\max }$ should follow adiabatically.

In addition, we can see that these solutions are stable by considering a perturbed cascade. If for any reason stirring becomes faster than catastrophic collisions, the velocities will increase and $r_{B}(r)$ will decrease until the catastrophic collision rate equals the new stirring rate. Similarly, if stirring becomes slower than catastrophic collisions, the velocities will slow and $r_{B}(r)$ will increase until collisions just balance stirring as long as $v(r) \geqslant v_{\mathrm{esc}}(r)$. The timescale for bodies smaller than some size $r$ to relax to this solution should be of order a few catastrophic collision times for size $r$ bodies.

We frame our discussion of the solutions via the velocity stirring/damping equilibria listed in Section 2.3. They suggest that given a disk in which a single $r_{B}(r)$ power-law relation applies to all bodies, and in which cooling has had time to 
operate, up to three velocity regimes occur. ${ }^{7}$ First, the largest bodies, which are stirred viscously and damped by dynamical friction, have velocities that are below their escape velocities but that increase with decreasing size according to Equation (26). At the size for which the bodies' velocity equals their escape velocity, dynamical friction can no longer cool efficiently and the second regime begins. Bodies slightly smaller than this first transition size have velocities faster than both their own escape velocities and the velocity of the largest bodies in the disk. In this regime stirring proceeds according to Equation (10) and damping proceeds by collisions. Third, if the cascade includes sufficiently small bodies, we expect for $p>0$ that the smallest bodies' velocities fall below the velocity of the largest body. In this regime stirring proceeds according to Equation (9) and collisional damping continues. While we would expect the power-law breaks associated with transitions between regimes will produce waves in the size and velocity distributions, we also expect that, on average, the sizes and velocities in each regime will be consistent with the $q$ and $p$ values we find. We discuss these three regimes-first, $v(r)<v_{\mathrm{esc}}(r)$; second, $v(r)>v_{\mathrm{esc}}(r)$ and $v(R)<v(r)$; third, $v(r)>v_{\text {esc }}(r)$ and $v(R)>v(r)$-in turn below.

The regime containing the largest bodies of sizes $r>r_{\max }$ is simplest. Because its bodies are not part of the cascade, we cannot constrain their size distribution except to require that it satisfy the conditions for Equation (10) to hold. Instead we expect that their size distribution $N(r) \propto r^{1-q^{\prime}}$ has not changed since their formation. Regardless of whether the bodies are gravity- or strength-dominated, Equation (26) gives

$$
\begin{array}{lll}
p=-3 / 2, & 1<q<7 & v(s)<v(r) \\
p=-3 / 4, & 1<q<7 & v(s)>v(r) .
\end{array}
$$

The remaining two velocity regimes, $v(r)<v(R)$ and $v(r)>v(R)$, may support cascades. We consider cascades with four different categories of $r_{B}(r)$ relations characterized by: (1) whether the bodies are gravity- or strength-dominated and (2) whether the bullet-target size ratio is close enough to unity for catastrophic collisions to provide effective cooling.

First we assume cooling by catastrophic collisions. This case requires a cascade. In the gravity regime, we solve Equations (6) and (20) simultaneously to get

$$
\begin{array}{llll}
p=\frac{17-\sqrt{241}}{4}, & q=\frac{\sqrt{241}-9}{2} & \text { for } & \alpha=\frac{4-p}{3} \\
p=\frac{11-\sqrt{85}}{4}, & q=\frac{\sqrt{85}-3}{2} & \text { for } \quad \begin{array}{l}
\alpha=\frac{5-2 p}{3} \\
v(R)>v(r)
\end{array}
\end{array}
$$

$$
\begin{aligned}
& p=\frac{31-\sqrt{865}}{8}, \quad q=\frac{\sqrt{865}-23}{2} \text { for } \alpha=\frac{4-p}{3} \\
& p=\frac{1}{4}, \quad q=3 \quad \text { for } \begin{array}{l}
\alpha=\frac{5-2 p}{3} \\
v(R)<v(r) .
\end{array}
\end{aligned}
$$

\footnotetext{
7 If significant external stirring has occurred, not all of these three regimes may occur. For example, if all the bodies in the disk have velocities larger than their own escape velocities, dynamical friction will never be important.
}

This implies

$$
\begin{array}{ll}
0.37<p<0.45 & \\
3.26>q>3.11 & \\
0.20<p<1 / 4 & \\
3.21>q>3 &
\end{array}
$$

In the strength regime, we likewise solve Equations (8) and (20) together for

$$
\begin{aligned}
& p=\frac{9+\gamma-\sqrt{69+6 \gamma+\gamma^{2}}}{4}, \\
& q=\frac{-1-\gamma+\sqrt{69+6 \gamma+\gamma^{2}}}{2} \quad v(R)>v(r) \\
& p=\frac{15+2 \gamma-\sqrt{201+36 \gamma+4 \gamma^{2}}}{8}, \\
& q=\frac{-7-2 \gamma+\sqrt{201+36 \gamma+4 \gamma^{2}}}{2} \quad v(R)<v(r) .
\end{aligned}
$$

For the range $-1 / 2<\gamma \leqslant 0$, these give

$$
\begin{array}{ll}
0.090<p \leqslant 0.17 & \\
3.82>q \geqslant 3.65 & \\
0.054<p \leqslant 0.10 & \\
3.78>q \geqslant 3.59 & v(R)<v(r) .
\end{array}
$$

Now we assume catastrophic collisions cannot damp the velocities significantly, so that the cooling timescale is the time it takes for a given body to collide with a total mass equal to its own. We also assume the lifetime of the disk is longer than this cooling timescale for all bodies with $v(r)>v_{\mathrm{esc}}$. In the gravity regime, these bodies all participate in the cascade: $v\left(r_{\max }\right) \sim v_{\text {esc }}\left(r_{\max }\right)$. Their steady-state sizes and velocities should follow from Equations (6) and (23). When $\alpha=(4-p) / 3$ this gives

$$
\begin{array}{llr}
p=\frac{6-\sqrt{34}}{2}, & q=9-\sqrt{34} & \text { for } \quad \begin{aligned}
& \alpha=\frac{4-p}{3} \\
& v(R)>v(r)
\end{aligned} \\
p=\frac{6-\sqrt{34}}{4}, & q=9-\sqrt{34} & \text { for } \quad \alpha=\frac{4-p}{3}
\end{array}
$$

We find, however, that no solution with $p \geqslant 0$ and $q \leqslant 4$ is possible when $\alpha=(5-2 p) / 3$. It turns out $q>4$ is also impossible in the gravity regime. If $q>4$, Equation (6) implies $p>3 / 2$, and since $v_{\mathrm{esc}}(r) \propto r$, having $p>1$ means that $v(r)$ will fall below $v_{\text {esc }}(r)$ at some $r$, stopping the cascade. Then the maximum $\alpha$ allowed must lie between $(4-p) / 3$ and $(5-2 p) / 3$. To find this limiting value, we recast Equations (4) and (5) as

$$
\rho r^{3} v_{\mathrm{esc}}^{\beta}(r) \sim \rho r_{B}^{3} v^{\beta}(r) \quad \longrightarrow \quad \alpha=1+\beta(1-p) / 3,
$$


where $1<\beta<2$. With Equations (3) and (23), this gives

$$
\begin{array}{ll}
p=\frac{3}{\beta} \pm \frac{\sqrt{36-6 \beta+4 \beta^{2}}}{2 \beta} & v(R)>v(r) \\
p=\frac{12+\beta}{4 \beta} \pm \frac{\sqrt{144+12 \beta+9 \beta^{2}}}{4 \beta} & v(R)<v(r) .
\end{array}
$$

A look at the zeros of $d p / d \beta$ shows that $p$ is monotonic for the relevant $\beta$, so the limiting $\alpha$ and $\beta$ should occur at a limiting value of $p$. For gravity-dominated bodies, $0 \leqslant p \leqslant 1$ as discussed above; for both $v(R)>v(r)$ and $v(R)<v(r)$, the only $\beta$ between 1 and 2 that satisfies $p=0$ or $p=1$ is $\beta=3 / 2$ at $p=0$. When $p=0$, Equation (23) gives $q=3$. Then the allowed $p, q$ in the gravity regime are

$$
\begin{aligned}
& 0 \leqslant p<0.085 \\
& 3 \leqslant q<3.17 \\
& 0 \leqslant p<0.042 \\
& 3 \leqslant q<3.17
\end{aligned}
$$

In the strength regime, not all of the bodies with $v(r)>v_{\mathrm{esc}}(r)$ can participate in the cascade. For those in the cascade, we first assume $q \leqslant 4$ and solve Equations (8) and (23) simultaneously. This gives

$$
\begin{aligned}
& p=\frac{4+\gamma-\sqrt{4+16 \gamma+\gamma^{2}}}{4} \\
& q=\frac{10+\gamma-\sqrt{4+16 \gamma+\gamma^{2}}}{2} \quad v(R)>v(r) \\
& p=\frac{5+\gamma-\sqrt{19+14 \gamma+\gamma^{2}}}{4} \\
& q=8+\gamma-\sqrt{19+14 \gamma+\gamma^{2}} \quad v(R)<v(r)
\end{aligned}
$$

When $\gamma<0$, the only real solutions to Equation (41) have $q>4$, which is inconsistent. The allowed ranges in $p, q$ are

$$
\begin{array}{rlrl}
p & =1 / 2 & v(R)>v(r) \\
q & =4 & \gamma=0 \\
1 / 4>p \geqslant 0.16 & v(R)<v(r) \\
4>q>3.64 & -1 / 2<\gamma \leqslant 0 .
\end{array}
$$

The $q>4$ which arose above when $v(R)>v(r)$ and $\gamma<0$ suggests that we look for a solution where the smallest bodies in the system dominate the collisional damping, that is, a solution using Equation (24) instead of Equation (23). Indeed, Equations (8) and (24) together give

$$
p=1 / 2, q=\frac{20+\gamma}{5+\gamma} \quad v(R)>v(r)
$$

and, for $-1 / 2<\gamma \leqslant 0$,

$$
\begin{aligned}
& p=1 / 2 \\
& 13 / 3>q>4
\end{aligned} \quad v(R)>v(r) .
$$

For bodies with $v(r)>v_{\text {esc }}(r)$ but $r>r_{\text {max }}$ - those not in the cascade - the primordial size distribution $q^{\prime}$ applies. As long as $q^{\prime}$ satisfies the conditions on Equation (9), the velocities follow from this and Equation (23) if $q^{\prime}<4$ or Equation (24) if $q^{\prime}>4$.

Finally, if the collisional cooling timescale is shorter than the age of the cascade, the velocity distribution will be shallower than predicted in the relevant regime above. How much shallower depends on particulars of the stirring timescale and the energy loss per collision. For example, if the kinetic energy lost in a catastrophic collision is so small that the kinetic energy retained by the fragments is larger than the energy they gain via stirring in one collision time, $p$ will instead depend on exactly how much energy is lost in an average collision. In turn the energy loss per collision depends heavily on the bodies' internal structure, which is very poorly constrained (Leinhardt et al. 2008, and references therein). We will not discuss this uncooled regime in detail here.

In the above we neglected any inward orbital drift caused by the energy loss in inelastic collisions. Since smaller bodies collide more frequently, and since the velocities are sizedependent, we might expect the orbital drift rates to be dependent on body size. Indeed, we can estimate the maximum drift rates by assuming that over one velocity damping timescale, the fractional orbital energy lost by a body of size $r$ is of order $v(r) /$ [orbital velocity]. Using Equations (12), (13), (20), and (23), this gives

orbital drift $\sim \frac{N\left(r_{B}(r)\right) r^{2} \Omega}{\text { area }} \frac{v(r)}{v_{\text {orbital }}} \propto \begin{cases}r^{-p} & v(R)>v(r) \\ r^{-3 p} & v(R)<v(r)\end{cases}$

when catastrophic collisions provide significant damping and

$$
\text { orbital drift } \sim \frac{N(r) r^{2} \Omega}{\text { area }} \frac{v(r)}{v_{\text {orbital }}} \propto \begin{cases}r^{-p} & v(R)>v(r) \\ r^{-3 p} & v(R)<v(r)\end{cases}
$$

when they do not. However, this orbital drift significantly affects the collisional cascade's progress only if the rate of relative drift between bullets and targets is faster than the catastrophic collision rate. If catastrophic collisions provide effective damping, the bullets and targets must differ in size by at most order unity. In this case we expect no significant relative drift because the power of $r$ in the drift rate is always less than unity. If catastrophic collisions do not provide effective damping, the damping rate is much slower than the catastrophic collision rate, so again we expect no significant relative drift on the catastrophic disruption timescale.

Our results for $p$ and $q$ in all the velocity and strength law regimes discussed in this work are summarized in Table 1. Note that all the size distributions are steeper than those that obtain when fixed velocities are used $(p=0)$; these are $3.14>q>2.88$ for the gravity regime (Pan \& Sari 2005) and $3.72>q \geqslant 3.5$ for the strength regime. The steepening is certainly consistent with smaller velocities for smaller bodies: lower velocities mean larger bullets are needed to break a target of a given size; an increase in bullet size corresponds to a decrease in the number of bullets for $q>0$; and a steeper size distribution offsets this decrease. Table 1 also confirms that our assumption $p<1$ of Section 2.1 is self-consistent.

\section{COMPARISON WITH NUMERICAL SIMULATIONS}

To test the analytic results above we used a numerical cascade simulation based on the coagulation code of Schlichting \& 
Table 1

Summary of Velocity Power Laws $p$ and Size Power Laws $q$ in Steady-state

\begin{tabular}{|c|c|c|c|c|c|}
\hline & & $\begin{array}{l}\text { Damping } \\
\text { Mechanism }\end{array}$ & $v(R)>v(r)$ & $v(R)<v(r)$ & References \\
\hline \multirow{7}{*}{$\begin{array}{l}v(r)>v_{\mathrm{esc}}(r) \text { : } \\
\text { includes all } \\
\text { bodies in } \\
\text { cascade }\end{array}$} & \multirow[t]{4}{*}{ Gravity regime } & Catastrophic & $0.37<p<0.45$ & $0.20<p<1 / 4$ & Equations (30), (31) \\
\hline & & collisions & $3.26>q>3.11$ & $3.21>q>3$ & \\
\hline & & Collisions with & $0 \leqslant p<0.085$ & $0 \leqslant p<0.042$ & Equations (39), (40) \\
\hline & & $\begin{array}{l}\text { equal-sized } \\
\text { bodies }\end{array}$ & $3 \leqslant q<3.17$ & $3 \leqslant q<3.17$ & \\
\hline & \multirow[t]{3}{*}{ Strength regime } & $\begin{array}{c}\text { Catastrophic } \\
\text { collisions }\end{array}$ & $\begin{array}{r}0.090<p \leqslant 0.17 \\
3.82>q \geqslant 3.65\end{array}$ & $\begin{array}{r}0.054<p \leqslant 0.10 \\
3.78>q \geqslant 3.59\end{array}$ & Equations (34), (35) \\
\hline & & $\begin{array}{l}\text { Collisions with } \\
\text { equal-sized } \\
\text { bodies }\end{array}$ & $\begin{array}{l}p=1 / 2 \\
q=4\end{array}$ & $\begin{array}{r}1 / 4>p>0.16 \\
4>q \geqslant 3.64\end{array}$ & Equations (44), (46) \\
\hline & & $\begin{array}{l}\text { Collisions with } \\
\text { smallest bodies }\end{array}$ & $\begin{array}{c}p=1 / 2 \\
13 / 3>q \geqslant 4\end{array}$ & $\cdots$ & Equation (48) \\
\hline $\begin{array}{l}v(r)<v_{\mathrm{esc}}(r): \\
\text { bodies too } \\
\text { large for } \\
\text { cascade }\end{array}$ & $\begin{array}{l}\text { Gravity or } \\
\text { strength } \\
\text { regime }\end{array}$ & $\begin{array}{l}\text { Dynamical } \\
\text { friction }^{\mathrm{a}}\end{array}$ & $\begin{array}{l}\cdots \\
\cdots\end{array}$ & $\begin{array}{c}p=-3 / 2 \text { or } p=-3 / 4 \\
1<q<7\end{array}$ & Equation (27) \\
\hline
\end{tabular}

Note. ${ }^{\mathrm{a}} p=-3 / 2$ applies when $v_{\text {esc }}(r)>v(r)>v(s) ; p=-3 / 4$ applies when $v(r)<v(s)<v_{\text {esc }}(r)$. Here $v(s)$ is the velocity of the bodies providing the dynamical friction.

Sari (2011). Because our goal here is to study the dominant physical processes in the cascade - viscous stirring, collisional and dynamical friction damping, and mass transfer from larger to smaller body sizes-we neglect factors of order unity in the stirring and damping rates. We study a single belt of bodies orbiting in an annulus about a much more massive star. We take the initial total mass in bodies to be about $1 M_{\text {Earth }}$, and we assume the bodies have bulk density $1 \mathrm{~g} / \mathrm{cc}$ and follow the mass and velocity evolution of bodies with radii ranging from $1 \mathrm{~m}$ to $3000 \mathrm{~km}$, a few times the size of Pluto.

As a first test of our velocity evolution theory, we artificially fix the size spectrum in the simulations and allow only the velocities to evolve. In Figure 1 we show as an example the test results with strength-dominated $\gamma=0$ bodies and a fixed $q=3.6$ size spectrum. Since we do not allow for catastrophic collisions in this run and since we fix the size spectrum at $q<4$, the collisional damping is dominated by collisions between similarly sized bodies as given in Equation (12). The resulting steady-state velocities obey Equation (23), which for $q=3.6$ means $p=0.3$ if $v(R)>v(r)$ and $p=0.15$ if $v(R)<v(r)$. We expect the velocities for large bodies with velocities below their own escape velocities to follow Equation (26). Our simulations agree well with these numbers.

Similarly, we test our mass cascade implementation by artificially fixing the velocity as a function of size and allowing only the size distribution to evolve. Figure 2 shows the results of a test with strength-dominated $\gamma=0$ bodies and velocities fixed to a broken power law with $p=1 / 4, p=1 / 8$. For these Equation (8) gives $q=3.73, q=3.61$; these agree well on average with our simulations. Our simulations also show waves as mentioned in Section 3; these are induced by the break in the velocity distribution as well as the artificial "breaks" in the mass power law created by the finite range of body sizes in our simulations.

Finally, we allow both the size and velocity distributions to evolve in the simulations. Figure 3 shows an example again using strength-dominated bodies with $\gamma=0$. We assumed in this run that the collisional damping of the velocity dispersion is dominated by collisions between like-sized bodies (see
Equation (12)). This criterion applies when catastrophic collisions do not damp the velocity dispersion significantly, which may occur for small bullet-to-target ratios. Here the steady-state solution of Equation (41) applies, and $\gamma=0$ implies $p=1 / 2$, $q=4$ when $v(R)>v(r)$ and $p=0.16, q=3.64$ when $v(R)<v(r)$. Again, these agree well with our simulations on average in each of the three different velocity regimes.

This model and the results shown in Figure 3 may, for example, apply at the end of protoplanetary growth in a planetesimal disk. Initially, the velocity dispersion is so small that collisions lead to growth. As the largest bodies- "protoplanets"-grow, they continue to excite the small planetesimals' velocity dispersion; their velocities grow on the same timescale as the large protoplanets' sizes (for a comprehensive description of this growth phase, see Schlichting \& Sari 2011). Once the system reaches an age comparable to the small planetesimals' collision time, but before collisions become destructive, the balance between gravitational stirring and collisional damping determines the planetesimals' velocity dispersion. This phase is similar to the situation shown in Figure 1, but with a mass spectrum that continues to evolve due to planetesimal accretion. Finally, the planetesimals' velocity dispersion is excited sufficiently above their escape velocities that destructive collisions set in. This stage is shown in Figure 3. The mass spectrum now no longer reflects the growth history; instead it is determined by the collisional evolution.

\section{SUMMARY}

We have found self-consistent steady-state solutions for the velocity function and size distribution of collisional cascades in the super-Hill regime. These solutions occur when mass conservation is satisfied and when viscous stirring balances velocity damping. Three kinds of velocity equilibrium may occur. For the biggest bodies, which have velocities slower than their escape velocities, viscous stirring and dynamical friction balance. These bodies' velocities increase with decreasing size until the size at which the velocity and escape velocity are equal. Since dynamical friction is inefficient for bodies with velocities 

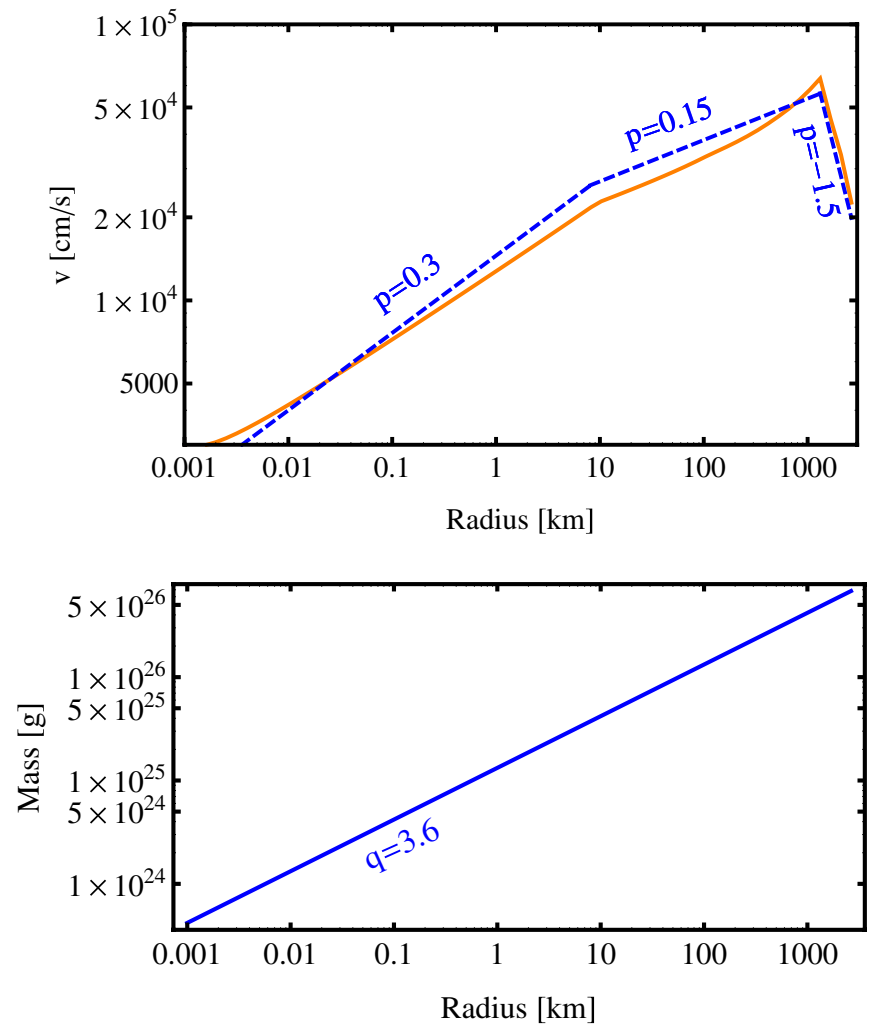

Figure 1. Comparison between analytic results (dashed blue line) and simulations (solid orange curve) for steady-state velocities in a system of strengthdominated bodies with $\gamma=0$. The top panel shows the mass in a given logarithmic mass bin as a function of radius, which in this run is fixed with $q=3.6$; the bottom panel shows the simulations and analytic results for the velocities. Since we do not allow for catastrophic collisions in this run and since we fix the size spectrum at $q<4$, the collisional damping is dominated by collisions between similarly sized bodies as given in Equation (12). There is good agreement in each of three velocity regimes. The smallest bodies, which have velocities greater than their own escape velocities but less than $v(R)$, follow $p=0.3$ (see Equation (23)). Larger bodies still small enough to have velocities larger than their own escape velocities, but which have velocities greater than $v(R)$, follow $p=0.15$ (see Equation (23)). Finally, the largest bodies have $p^{\prime}=-3 / 2$ because they are subject to dynamical friction by small bodies with velocity dispersion $v(r)<v(R)$ (see Equation (26)).

(A color version of this figure is available in the online journal.)

faster than their escape velocities, stirring balances damping by direct collisions for all smaller bodies. Bodies just smaller than this first transition size have velocities faster than both their escape velocities and the velocity of the largest bodies in the system. A second transition occurs at the body size whose velocity equals that of the largest bodies in the system. Bodies smaller than this second transition have velocities slower than the largest bodies in the system, so their stirring requires a different cross-section. The resulting size distributions for the gravity- and strength-dominated regimes are steeper than the ones expected with size-independent velocities. We find good agreement between the predictions of our theory and the results of our numerical simulations.

To our knowledge, previous analytic treatments of collisional cascades have not considered velocity stirring or damping. Wyatt (2008) and Kennedy \& Wyatt (2010) study disks in which the cascade start time depends on orbital radius because the large bodies needed to excite the velocity dispersion and initiate a cascade take longer to accrete at larger orbital radii. However, they do not consider the effects of stirring or damping on colliding bodies' velocities as the cascade proceeds.
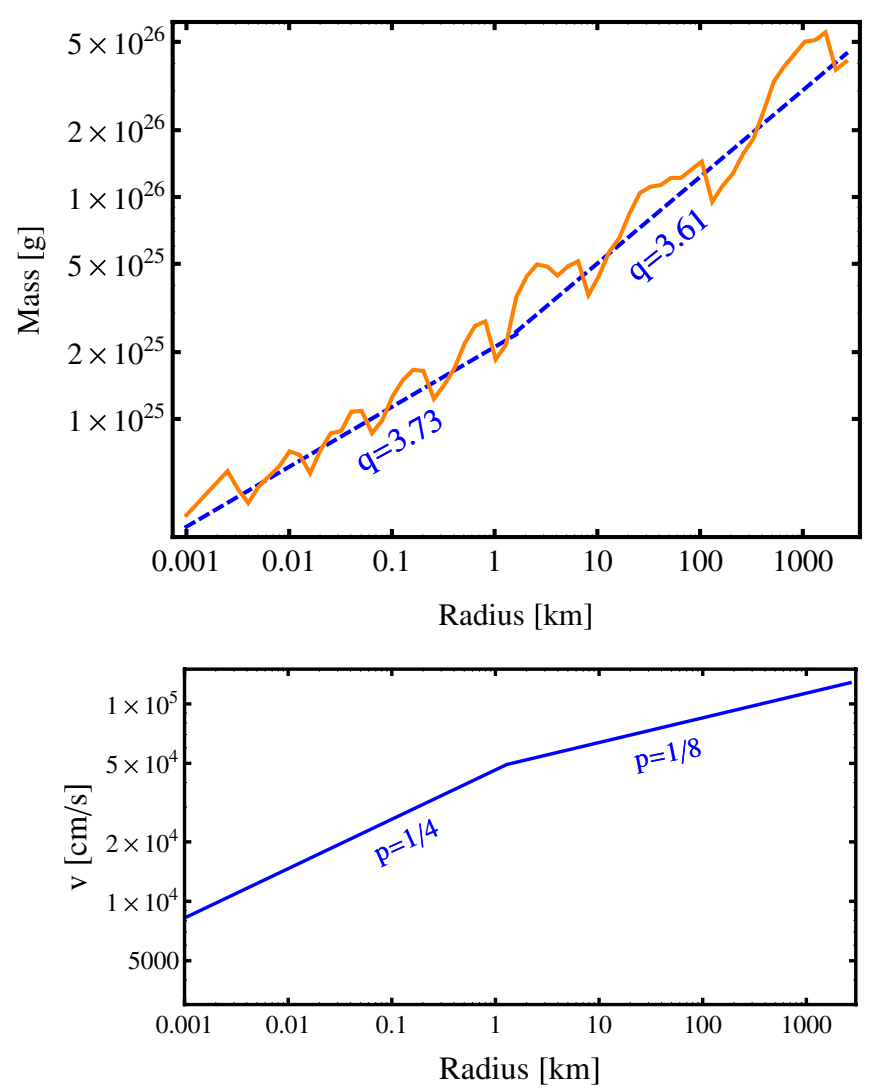

Figure 2. Comparison between analytic results (dashed blue line) and simulations (solid orange curve) in a steady-state system of strength-dominated bodies with $\gamma=0$ and fixed velocity distribution. The top panel shows the resulting mass spectrum plotted as mass in a given logarithmic mass bin as a function of radius (solid orange curve) and the corresponding analytic results (dashed blue line); the bottom panel shows the fixed velocity distribution. We begin the simulations with an initial size distribution $q=3.6$, which evolved to the $q=3.61$ for $v(r)>v(R)$ and $q=3.73$ for $v(r)<v(R)$ as expected from Equation (8) with $p=1 / 4, p=1 / 8$. Waves are clearly visible as oscillations in the steady-state mass spectrum. We note here that the wavelength of the waves changes as one transitions from the $p=1 / 4$ to the $p=1 / 8$ velocity distribution. This change in wavelength reflects the velocity dependence in the bullet-to-target ratio.

(A color version of this figure is available in the online journal.)

Kenyon \& Bromley (2008) do account for simultaneous velocity and size spectrum evolution in their numerical coagulation/fragmentation code. Our results here are not directly comparable to theirs because the largest bodies in their simulations continue to accrete while their collisional cascades operate. We plan to extend and modify our calculations to enable comparison with their findings. Other areas for future investigation include incorporating velocity stirring and damping into collisional cascades covering both the gravity and strength regimes as well as the waves induced in the size and velocity power laws due to transitions between regimes. A good knowledge of the size and velocity distributions will also allow us to predict observables such as the dust production rate as a function of time and the scale height of the disk as a function of size or, for the smallest bodies, observing wavelength. For the smallest dust particles a complete velocity treatment must account for excitation by radiation effects such as radiation pressure and Poynting-Robertson drag. This calculation would be an analytic extension of the work of Thébault (2009), who used numerical simulations to study the balance between radiation pressure and collisions in a disk with a fixed size distribution. There may also 

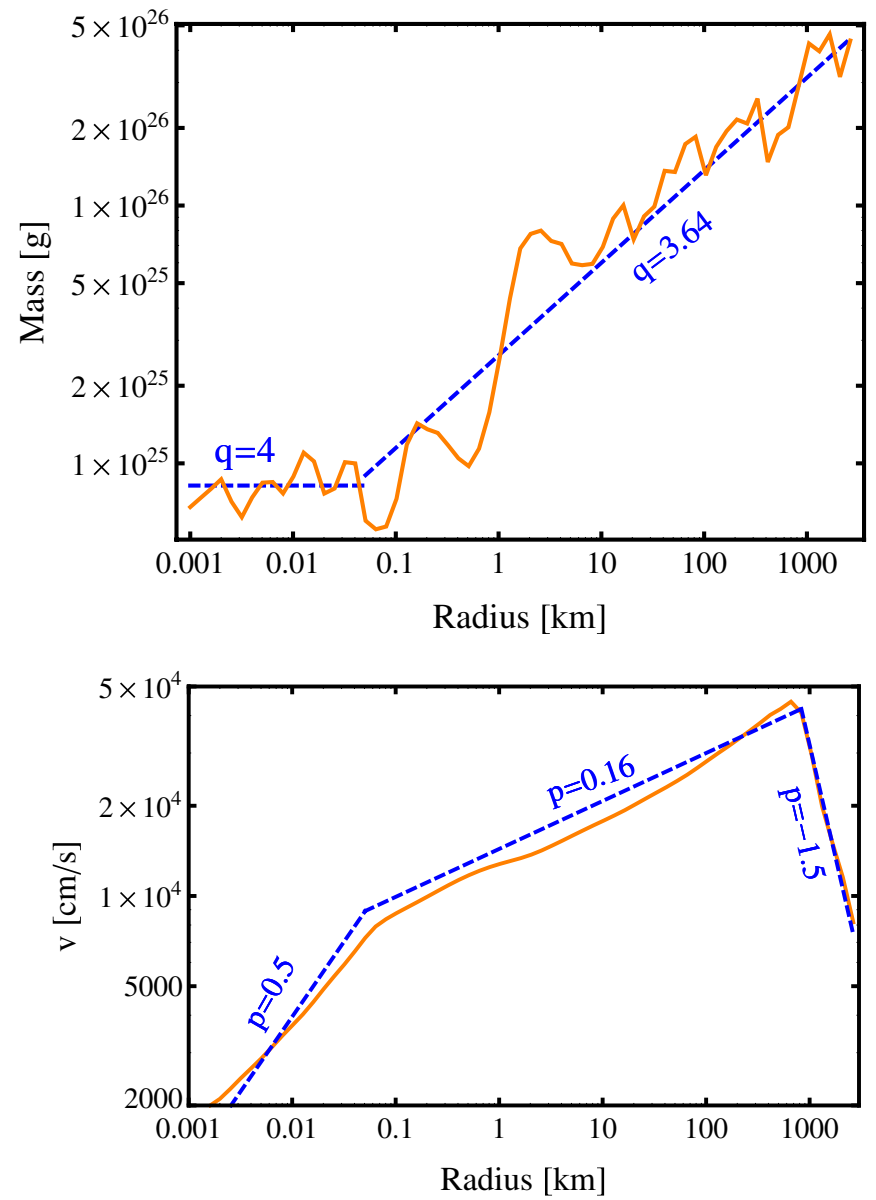

Figure 3. Comparison between analytic results (dashed blue line) and simulations (solid orange curve) in which the size distributions and velocities are both evolved together. The top panel shows the mass spectrum; the bottom panel shows the velocity distribution. In this run we assumed that the collisional damping of the velocity dispersion is dominated by collisions between like-sized bodies (see Equation (12)). This damping criterion applies when catastrophic collisions do not damp the velocity dispersion significantly, which may occur for small bullet-to-target ratios. The agreement between the simulations and our analytic results in Equations (41) and (26) is good on average for the $v(R)<v(r)$ and $v(R)>v(r)$ regimes in both the mass and velocity plots as well as for the $v(r)<v_{\text {esc }}(r)$ regime (see the caption of Figure 1 for a description of the regimes). Waves due to both the transition between $v(R)>v(r)$ and $v(R)<v(r)$ and the ends of our simulation range are again visible in the data.

(A color version of this figure is available in the online journal.)

be some separation in orbital radius between the large planetesimals and the dust due to radiation drag as well as the relative drift described by Equations (49) and (50).

Ongoing surveys of the Kuiper and asteroid belts provide observational size distribution and velocity data to which we can compare our results. Kuiper belt surveys indicate that its size spectrum follows a broken power law whose break falls at a body size of several tens of kilometers (Bernstein et al. 2004; Fraser 2009); this break is interpreted as the top of a collisional cascade. Typical Kuiper belt velocities are about $1 \mathrm{~km} \mathrm{~s}^{-1}$, of order 30 times larger than the escape velocities from the largest bodies in the cascade, so the typical bullet/target size ratio is far from unity. Then cooling by catastrophic collisions should be ineffective. Also, the timescales for the observed Kuiper belt objects (KBOs) to collide with bodies of equal size are longer than the age of the solar system. These KBOs have therefore not had time to cool; we would expect their average velocities should be very similar to those of the primordial KBOs. Indeed, small KBOs' eccentricities and inclinations show no significant trends with size. As for the size distribution, assuming the break exists, surveys find a range of size distributions $1.9<q<3.9$ for KBOs smaller than the break size (Bernstein et al. 2004; Fraser et al. 2008; Fraser 2009; Schlichting et al. 2009; Bianco et al. 2010). This is consistent with the $2.88<q<3.14$ we expect if $p \simeq 0$ but not strongly constraining. In the asteroid belt, typical relative velocities of $\sim 5 \mathrm{~km} \mathrm{~s}^{-1}$ suggest catastrophic collisions are likewise ineffective at cooling. Surveys of the asteroid belt indicate a size distribution of $q \simeq 3.5$ for large bodies of $H$ magnitude smaller than about 15 , or size larger than $\sim 1 \mathrm{~km}$ (Gladman et al. 2009, and references therein). For smaller bodies, however, the slope becomes shallower; different surveys report slopes ranging from $q=2$ to $q=2.8$ (Ivezić et al. 2001; Yoshida et al. 2003; Yoshida \& Nakamura 2007; Wiegert et al. 2007). While the overall size distribution slope is roughly consistent with the expected $2.88<q<3.14$, we would predict that the average slope steepen for bodies smaller than about $100 \mathrm{~m}$ in size. Still, our theory alone suggests several possible causes for waves that might explain the observed break and its location. This again makes strong constraints difficult without further data on smaller bodies.

We look forward to future observations of smaller KBOs and asteroids whose cooling time may be shorter than the belts' lifetimes and which will provide a longer size baseline with which to compare our theory. Future survey results of this kind will provide more stringent tests of our results and may shed light on the catastrophic collision process in our solar system. In particular, measurements of the slopes of the size and velocity distributions would provide a direct probe of the bodies' strengths. Similarly, observations of debris disk scale heights as a function of wavelength at millimeter wavelengths, for example with the Atacama Large Millimeter/Submillimeter Array, would provide direct tests of our velocity power laws as well as constraints on the internal strengths of pebble-sized particles in those disks.

It is a pleasure to thank Re'em Sari for helpful discussions. For H.S. support for this work was provided by NASA through Hubble Fellowship Grant HST-HF-51281.01-A awarded by the Space Telescope Science Institute, which is operated by the Association of Universities for Research in Astronomy, Inc., for NASA, under contact NAS 5-26555. M.P. was partially supported by NSF Grant AST-0909210.

\section{REFERENCES}

Belyaev, M. A., \& Rafikov, R. R. 2011, Icarus, 214, 179

Benz, W., \& Asphaug, E. 1999, Icarus, 142, 5

Bernstein, G. M., Trilling, D. E., Allen, R. L., et al. 2004, AJ, 128, 1364

Bianco, F. B., Zhang, Z.-W., Lehner, M. J., et al. 2010, AJ, 139, 1499

Campo Bagatin, A., Cellino, A., Davis, D. R., Farinella, P., \& Paolicchi, P. 1994, Planet. Space Sci., 42, 1079

Dohnanyi, J. S. 1969, J. Geophys. Res., 74, 2531

Durda, D. D., \& Dermott, S. F. 1997, Icarus, 130, 140

Fraser, W. C. 2009, ApJ, 706, 119

Fraser, W. C., \& Kavelaars, J. J. 2009, AJ, 137, 72

Fraser, W. C., Kavelaars, J. J., Holman, M. J., et al. 2008, Icarus, 195, 827

Gladman, B. J., Davis, D. R., Neese, C., et al. 2009, Icarus, 202, 104

Goldreich, P. M., Lithwick, Y., \& Sari, R. 2004, ARA\&A, 42, 549

Ivezić, Ž., Tabachnik, S., Rafikov, R., et al. 2001, AJ, 122, 2749

Jutzi, M., Michel, P., Benz, W., \& Richardson, D. C. 2010, Icarus, 207, 54

Kennedy, G. M., \& Wyatt, M. C. 2010, MNRAS, 405, 1253

Kenyon, S. J., \& Bromley, B. C. 2004, AJ, 128, 1916

Kenyon, S. J., \& Bromley, B. C. 2008, ApJS, 179, 451

Krivov, A. V., Sremčević, M., \& Spahn, F. 2005, Icarus, 174, 105 
Leinhardt, Z. M., Stewart, S. T., \& Schultz, P. H. 2008, in Physical Effects of Collisions in the Kuiper Belt, ed. M. A. Barucci, H. Boehnhardt, D. P. Cruikshank, A. Morbidelli, \& R. Dotson (Tucson, AZ: Univ. Arizona Press), 195

Löhne, T., Krivov, A. V., \& Rodmann, J. 2008, ApJ, 673, 1123

O’Brien, D. P., \& Greenberg, R. 2003, Icarus, 164, 334

O'Brien, D. P., \& Greenberg, R. 2005, Icarus, 178, 179

Pan, M., \& Sari, R. 2005, Icarus, 173, 342

Schlichting, H. E., Ofek, E. O., Wenz, M., et al. 2009, Nature, 462, 895
Schlichting, H. E., \& Sari, R. 2011, ApJ, 728, 68

Stewart, S. T., \& Leinhardt, Z. M. 2009, ApJ, 691, L133

Tanaka, H., Inaba, S., \& Nakazawa, K. 1996, Icarus, 123, 450

Thébault, P. 2009, A\&A, 505, 1269

Wiegert, P., Balam, D., Moss, A., et al. 2007, AJ, 133, 1609

Williams, D. R., \& Wetherill, G. W. 1994, Icarus, 107, 117

Wyatt, M. C. 2008, ARA\&A, 46, 339

Yoshida, F., \& Nakamura, T. 2007, Planet. Space Sci., 55, 1113

Yoshida, F., Nakamura, T., Watanabe, J.-I., et al. 2003, PASJ, 55, 701 